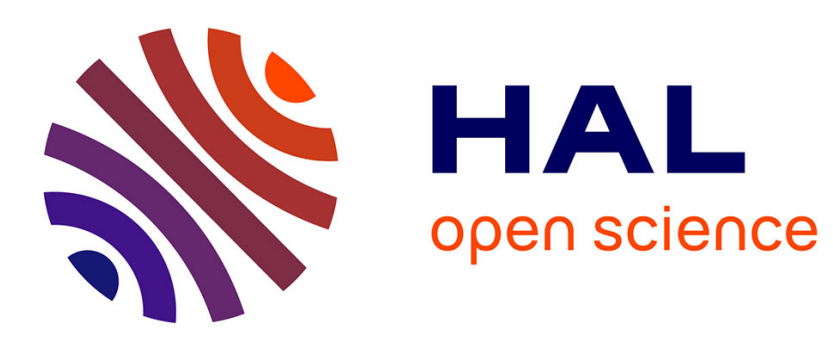

\title{
De l'appréhension des significations implicites : verbes factifs et contrefactifs
}

\author{
Edith Salès-Wuillemin
}

\section{To cite this version:}

Edith Salès-Wuillemin. De l'appréhension des significations implicites : verbes factifs et contrefactifs. Annee Psychologique, 1991, 91 (4), pp.581-602. halshs-00594271

\section{HAL Id: halshs-00594271}

https://shs.hal.science/halshs-00594271

Submitted on 19 May 2011

HAL is a multi-disciplinary open access archive for the deposit and dissemination of scientific research documents, whether they are published or not. The documents may come from teaching and research institutions in France or abroad, or from public or private research centers.
L'archive ouverte pluridisciplinaire HAL, est destinée au dépôt et à la diffusion de documents scientifiques de niveau recherche, publiés ou non, émanant des établissements d'enseignement et de recherche français ou étrangers, des laboratoires publics ou privés. 


\title{
Persée
}

http://www.persee.fr

\section{De l'appréhension des significations implicites : verbes factif $s$ et contrefactifs}

\author{
Edith Sales-Wuillemin \\ L'Année psychologique, Année 1991, Volume 91, Numéro 4 \\ p. $559-580$
}

\section{Voir l'article en ligne}

Résumé De nombreuses distinctions entre factifs, contre factifs, non-factifs ont été proposées sur la base de critères logiques ou linguistiques. Nous faisons l'hypothèse selon laquelle, dans les phrases contenant ces verbes suivis d'une proposition subordonnée, les critères logiques ou linguistiques ne sont pas les seuls éléments pris en compte par les sujets qui doivent juger de la vérité de l'événement contenu dans la proposition subordonnée. Entrent également en jeu des éléments extra-linguistiques comme la compétence de la source. Nous distinguons, dans l'usage indirect de ces verbes, deux sources différentes : le locuteur et l'énonciateur. Nous analysons l'appréhension, par des sujets, du présupposé véhiculé par des verbes factifs et contrefactifs, en faisant varier la compétence de chaque source. Les résultats mettent en évidence le fait que c'est la compétence de l'énonciateur qui joue un rôle déterminant, et ce audelà des critères linguistiques.

\section{Avertissement}

L'éditeur du site «PERSEE » - le Ministère de la jeunesse, de l'éducation nationale et de la recherche, Direction de l'enseignement supérieur, Sous-direction des bibliothèques et de la documentation - détient la propriété intellectuelle et les droits d'exploitation. A ce titre il est titulaire des droits d'auteur et du droit sui generis du producteur de bases de données sur ce site conformément à la loi n`98-536 du 1 er juillet 1998 relative aux bases de données.

Les oeuvres reproduites sur le site «PERSEE » sont protégées par les dispositions générales du Code de la propriété intellectuelle.

Droits et devoirs des utilisateurs

Pour un usage strictement privé, la simple reproduction du contenu de ce site est libre.

Pour un usage scientifique ou pédagogique, à des fins de recherches, d'enseignement ou de communication excluant toute exploitation commerciale, la reproduction et la communication au public du contenu de ce site sont autorisées, sous réserve que celles-ci servent d'illustration, ne soient pas substantielles et ne soient pas expressément limitées (plans ou photographies). La mention Le Ministère de la jeunesse, de l'éducation nationale et de la recherche, Direction de l'enseignement supérieur, Sous-direction des bibliothèques et de la documentation sur chaque reproduction tirée du site est obligatoire ainsi que le nom de la revue et- lorsqu'ils sont indiqués - le nom de l'auteur et la référence du document reproduit.

Toute autre reproduction ou communication au public, intégrale ou substantielle du contenu de ce site, par quelque procédé que ce soit, de l'éditeur original de l'oeuvre, de l'auteur et de ses ayants droit.

La reproduction et l'exploitation des photographies et des plans, y compris à des fins commerciales, doivent être autorisés par l'éditeur du site, Le Ministère de la jeunesse, de l'éducation nationale et de la recherche, Direction de l'enseignement supérieur, Sous-direction des bibliothèques et de la documentation (voir http://www.sup.adc.education.fr/bib/ ). La source et les crédits devront toujours être mentionnés. 
Groupe de Recherche sur la Parole

Université Paris VIII

UFR Psychologie pratique clinique et sociale ${ }^{1}$

\author{
DE L'APPREHENSION \\ DES SIGNIFICATIONS MPLICITES : \\ VERBES FACTIFS ET CONTREFACTIFS \\ par Edith Sales-Wuillemin
}

SUMMARY : Understanding implicit meanings : Factive and counter factive verbs.

Various distinctions between factive, counter factive, and non-factive verbs have been proposed, based on logical or linguistic criteria. Our hypothesis is that in sentences containing these types of verbs constructed awith completive clauses, linguistic criteria are not the only ones taken into account by the subject who is to judge whether the statement is true or not. Other elements, like the source's competence, interfere are also taken into account. Two different sources have been considered : speaker and communicator.

We examined how subjects grasped presupposed information in sentences containing factive or counter factive verbs. These sentences were constructed varying the competence of speaker and communicator. Results show that it is the competence of the communicator which is the more important, beyond linguistic criteria.

Key-words : factive verbs, non-factive verbs, counter factive verbs, presupposition, language comprehension.

\title{
INTRODUCTION
}

Ce que nous essaierons de mettre en évidence dans cet article c'est la façon dont, partant du constat selon lequel on ne peut rendre compte de la compréhension des énoncés uniquement en

1. 2, rue de la Liberté, 93526 Saint-Denis Cedex 02. 
termes sémantiques ou logiques, on passe de la vision d'un sens enfermé dans la langue d une conception incluant des facteurs extra-linguistiques. Ici la compréhension n'est plus traitée en termes de simple décodage, mais comme faisant appel à des mécanismes inférentiels fondés à la fois sur le contenu de l'énoncé et sur les savoirs des sujets. Dans ce contexte, nous nous proposons d'examiner l'appréhension des présupposés véhiculés par un support particulier : les verbes factifs et contrefactifs.

Notons tout d'abord que l'étude de ce type de verbes a donné lieu a deux courants de recherches que l'on peut schématiquement dénommer logico-linguistique et psychologique. Dans le premier cas, ces verbes ont été analysés en fonction de critères sémantiques ou logiques, dans le deuxième cas, on a été amené à étudier l'appréhension de ces verbes compte tenu de variables psychologiques, i.e. liées aux savoirs des sujets.

\section{LES ÉTUDES LOGICO-LINGUUSTIQUES}

Nous rappellerons simplement ici que, outre les verbes d'opinion, se répartissent au sein de deux principales catégories : 1) les verbes factifs (comme savoir, regretter, se douter, être content...) analysés par Kiparsky et Kiparsky (1970), qui ont pour caractéristique principale de poser une eroyance à l'égard d'un événement, et de présupposer la vérité/réalité de cet événement; et 2) les verbes contrefactifs (comme s'imaginer, prétendre...), étudiés par Harris (1974), qui posent une croyance à l'égard d'un événement et présupposent la fausselé de cet événement. A ces deux catégories il faut adjoindre, selon Kiparsky et Kiparsky (ibid.), la classe des non-factifs composée de verbes (comme croire, supposer, penser...) qui ont pour caractéristique de ne véhiculer aucun présupposéz

Ainsi, si nous comparons :

(1) "Pierre sait que Jean viendra " à

(2) "Pierre prétend que Jean viendra " et à

(3) "Pierre pense que Jean viendra ",

2. Zuber (1977) distingue quant à lui les Factifs émotifs (comme regretter, déplorer, être content de...) des Factifs non émotifs (comme se rendre compte de, savoir, prendre en considération...). 
nous remarquons que (1) pose que Pierre a une opinion quant à la venue de Jean et présuppose la vérité/réalité de cet événement, alors que (2) pose la même chose, mais véhicule en plus une information qui vient contredire la croyance de Pierre à l'égard de la venue de Jean, cette information présupposant que Jean ne viendra pas. L'énoncé (3) par contre ne véhicule pas d'informations quant à la venue de Jean.

Il est nécessaire toutefois, comme le propose R. Martin (1987), de distinguer, pour ce qui concerne les verbes factifs et non factifs, l'usage direct (c'est-à-dire lorsque le verbe est à la première personne du singulier du présent de l'indicatif, comme dans " je sais (crois) que... ") de l'usage oblique (c'est-à-dire lorsque le verbe est employé à une autre personne, comme dans " il sait (croit) que... ", ou bien lorsqu'il est utilisé à la première personne du singulier mais à un autre temps que l'indicatif présent, comme dans " je savais (croyais) que... ").

- Pour ce qui concerne l'usage direct du verbe faclif (" savoir que... ", R. Martin précise, conformément à ce que nous avons vu, que "Je sais que $p$ " présuppose la vérité de $p$. Par contre, l'auteur met en évidence le fait que l'usage oblique du verbe " savoir que " ne fonctionne pas tout à fait de la même façon car il met en jeu une hiérarchie des univers de croyance : "l'hétérounivers ", subordonné à l'univers du " je ", crée par là même ce que l'on pourrait appeler une cascade de présupposés.

Ainsi, si je dis "Il sait que Marie est là ", cela présuppose " $J e$ sais que Marie est là "(et par voie de conséquence, comme dans l'usage direct, la vérité de la proposition "Marie est là ").

De la même manière on aura " je savais que $p$ " et "Je ne savais pas que $p$ " qui présupposent " je sais que $p$ ".

- A propos de l'usage du verbe non factif " croire que ", Martin proposera une conception quelque peu divergente de celle évoquée par Kiparsky et Kiparsky (ibid.). En effet, selon ces auteurs, le verbe " croire ", comme tous les verbes de la catégorie des non-factifs, ne véhicule aucun présupposé. Selon R. Martin, le verbe " croire " a au contraire une caractéristique fondamentale, due à une différence dans son comportement selon que l'on considère l'usage direct ou l'usage oblique.

Ainsi, selon l'auteur, "dans son usage oblique, le verbe croire, comme tout verbe épistémique, implique deux univers de croyance, celui évoqué de la personne dont le locuteur décrit l'univers de croyance et celui sous-jacent du locuteur lui-même. 
L'hypothèse sera que dans le premier la valeur de $p$ tend vers le vrai et que dans l'autre elle tend vers le faux. Dans l'usage direct, c'est le propre univers du locuteur qui se trouve évoqué, et la valeur de vérité de $p$ y tendra vers le vrai " (p. 54).

En conséquence, selon R. Martin, dire " il croit que Marie va l'épouser " c'est laisser entendre que moi je ne le crois pas. De la même façon, dire " je croyais que j'étais malade " c'est laisser entendre qu'actuellement " je ne le crois plus".

Par contre, un énoncé tel que " je crois qu'il viendra ", aurait selon l'auteur un cinétisme naturel qui tendrait ${ }^{3}$ vers le vrai.

En guise de résumé des conceptions développées par R. Martin nous reprendrons le schéma, concernant les verbes factifs, contrefactifs, et le verbe non factif "dire ", qui fut proposé par l'auteur en 1983 (Martin, 1983, p. 98).

"Il dit que $p "-\frac{\mid p l^{4}}{? p \mathrm{~L}}$

"Il s'imagine que $p »-|p|$ $p \mathrm{~L}$

"Il sait que $p "-\mid \begin{aligned} & p \mathrm{l} \\ & -\mid \\ & p \mathrm{~L}\end{aligned}$

\section{LES ÉTUDES PSYCHOLOGIQUES}

Les études psychologiques partent du constat que l'on ne peut rendre compte de phrases contenant des présuppositions (ou entraînant des implications) uniquement en termes sémantiques ou logiques : il est nécessaire d'introduire des variables psychologiques relatives aux savoirs que les sujets possèdent sur le monde ${ }^{5}$.

3. Il s'agit bien ici de tendances, car la proposition introduite par * je crois que... * n'est jamais tenue à coup sûr pour vraie par le locuteur. Ainsi, sauf dans le cas de l'emploi d'une stratégie particulière, ce serait faire preuve de mauvaise foi (et contrevenir à la maxime de qualité décrite par H. P. Grice (1975)) que de dire " je crois que Marie est là " si l'on sait pertinemment que Marie est là. De la même manière, il apparaft incongru de placer dans le champ de croire que... une proposition qui ne peut être mise en doute, comme c'est le cas pour " je crois que j'ai mal (p. 57).

4. Où - - est équivalent à $p$ est vrai $(-\mid$ étant le symbole de l'assertion); I est équivalent a locuteur (ou énonciateur dans la terminologie de O. Ducrot (1980, p. 43)) ; et $\mathrm{L}$ est équivalent Locuteur (au sens de celui qui parle).

5. Pour une revue détaillée des travaux réalisés dans le cadre de la psycholinguistique cf. Day et Caron (1991). 
C'est dans cette perspective que se situent les travaux de Oléron et Legros qui dès 1977 analysent (à partir des diverses conceptions développées par Kiparsky et Kiparsky (1970) ; Karttunen (1971); Fillmore (1971); Harris (1974); Ducrot (1972)) l'appréhension, par des enfants de 4 et 7 ans de différentes catégories de verbes (factifs, non factifs, contrefactifs, implicatifs et implicatifs négatifs ) qui sont présentés dans leur usage oblique.

Les verbes implicatifs (comme " réussir ", " parvenir ", " se rappeler »...) ont été analysés par Karttunen (1971, 1973). Ces verbes ont pour caractéristique principale de se comporter comme des factifs lorsqu'ils sont à la forme affirmative (ils impliquent la vérité de la phrase complément). Par contre, lorsqu'ils sont à la forme négative, contrairement aux verbes présuppositionnels, la phrase complément est niée. Ce phénomène est dû au fait que l'implication (à l'inverse de la présupposition) ne résiste pas à la négation.

Cette expérimentation visait d'une part à tester la capacité des sujets à appréhender le présupposé ou l'implication véhiculés par les phrases présentées, et d'autre part à mettre en évidence Ies éléments sur lesquels se fondent les sujets pour saisir ce type d'implicite.

Les résultats montrent que l'appréhension des présuppositions véhiculées par l'un des verbes factif-affirmatif (" découvrir ") est conforme au modèle linguistique dès l'âge de 4 ans, le verbe "savoir " n'étant appréhendé qu'à partir de 7 ans. Alors que pour les autres types de verbes cela est variable et s'améliore avec l'âge.

Pour Lehalle et Jouen (1978) qui testent la compréhension de verbes présuppositionnels chez les élèves de 12 a 18 ans, la distinction entre posé et présupposé pour les verbes contrefactifs (" s'imaginer ", " se figurer ") n'apparait pas systématiquement même chez les élèves de terminale. En ce qui concerne les verbes factifs (" se douter ") la quasi-totalité des sujets les plus jeunes (12 ans) ne répondent pas conformément au présupposé ce résultat semblant s'améliorer avec l'âge. Par ailleurs, Mac Namara, Backer et Olson (1976), testant sur des enfants de 4 ans l'appréhension de trois types de verbes (" faire semblant ", " oublier " et " savoir " mis à la forme affirmative et négative, remarquent que la compréhension de "faire semblant 》 n'est correcte que lorsque ce verbe est à la forme négative. En 
ce qui concerne le verbe "oublier ", les enfants de cet âge sont capables d'appréhender le présupposé et l'implication que le verbe soit a la forme affirmative ou négative. Par contre, pour le verbe " savoir ", il semble qu'une forte proportion des enfants n'ait pas appréhendé le présupposé.

En ce qui concerne les verbes non factifs Oléron et Legros (op. cit.) n'obtiennent pas des résultats conformes au modèle linguistique, à savoir des réponses de doute, d'abstention ou d'ignorance. Les auteurs expliquent ces résultats en faisant référence à la difficulté que les enfants ont à produire spontanément de telles réponses.

Mais au-delà de ce fait, il est apparu que les enfants de 7 ans ont traité trois des verbes non factifs (souhaiter, craindre, et être impatient) et un des verbes factifs (être content) comme des verbes implicatifs. Ce qui s'expliquerait selon eux par le fait que les sujets n'ont pas appréhendé ces verbes en fonction de critères strictement linguistiques, mais également en fonction de critères psychologiques.

Dès lors, Oléron et Legros (1986) proposeront d'interpréter les résultats obtenus antérieurement en se référant aux savoirs que les sujets possèdent sur le monde. Plus précisément, les auteurs mettent l'accent sur le fait que c'est la connaissance des événements sur lesquels les sujets peuvent agir qui détermine leurs réponses. Ainsi si certains énoncés comme " P... n'était pas content de prêter ses jouets " ont entrâné une réponse négative à la question " Est-ce que P... a prêté ses jouets ?", c'est parce que pour les sujets, un enfant qui n'est pas content de prêter ses jouets ne les prête pas. Par contre, si d'autres énoncés comme "P... n'était pas content qu'il pleuve " ont suscité une réponse positive à la question "Est-ce qu'il pleuvait ? " (et ce bien que le verbe soit identique) cela s'explique de par le fait que les sujets sont conscients du fait que l'état psychologique ne peut entraîner, dans ce cas, de modifications en ce qui concerne l'état du monde (que P... soit content ou non qu'il pleuve, il n'en reste pas moins qu'il pleut).

A partir de cette analyse les auteurs proposent de répartir les verbes factifs en deux catégories : les factifs stricts et les factifs/implicatifs qui se comportent comme des factifs ou des implicatifs en fonction du contexte (cette distinction étant également applicable aux non-factifs).

Les factifs feraient référence à un état du monde indépendant 
du sujet, état qui n'est pas modifié par son état psychologique, qui peut être d'ordre cognitif (savoir, découvrir...) ou encore d'ordre appréciatif ou affectif (regretter, se réjouir...). Au contraire les implicatifs feraient référence à un état du monde dépendant du sujet (décider, choisir...). Par contre les factifs/ implicatifs se comporteraient comme des factifs ou comme des implicatifs en fonction du contexte (e.g. être content, être ennuyé...).

Les travaux de Oléron et Legros en mettant en évidence le rôle des savoirs des sujets dans l'interprétation de ce type de verbes permettent de dépasser les catégories établies sur des critères strictement linguistiques ou logico-linguistiques. Toutefois, s'ils établissent une relation entre le sujet interprétant et le langage, ils n'envisagent pas le rôle joué par le locuteur et la prise en compte de ce locuteur par le sujet.

Nous ferons ici l'hypothèse générale selon laquelle le support lexical et les savoirs relatifs à l'état du monde ne sont pas les seuls éléments qui soient pris en compte par le sujet lorsqu'il est amené à juger de la vérité de l'événement rapporté. Sont également prises en considération d'autres informations d'ordre extra-linguistique comme par exemple le degré de confiance attribué aux différentes sources mises en jeu dans l'énoncé.

En d'autres termes, nous partirons de l'hypothèse selon laquelle un énoncé ne peut être jugé isolément, il renvoie à :

1) une source (A) qui est le locuteur (i.e. celui qui parle) qui dans le cas des verbes factifs et contrefactifs se pose comme ayant un savoir avéré et est responsable du présupposé qu'il prend en charge. En conséquence, l'utilisation d'un verbe factif introduisant un fait ou un événement (appelons-le " y ") permet au locuteur de marquer lexicalement en langue la vérité/réalité de cet événement. L'utilisation d'un verbe contrefactif permettant à l'inverse de marquer la fausseté/ irréalité de l'événement en question;

2) une source (B) qui est l'énonciateur c'est-à-dire celui dont on parle qui est à l'origine de la croyance i.e. du posé ;

3) un sujet interprétant qui a des savoirs et compétences et qui attribue des savoirs et compétences au locuteur ainsi qu'à l'énonciateur : l'ensemble de ces informations étant utilisé lors du jugement relatif au degré de vérité/réalité de l'événement rapporté. 
Ces différentes remarques nous amènent à poser les hypothèses suivantes :

\section{HYPOTHËSES :}

H1 : Le degré de vérité/réalité attribué à l'événement " $y$ " contenu dans la proposition enchâssée sera directement dépendant de la compétence attribuée à la source : il devrait être renforcé si la source est compétente et atténué dans le cas contraire.

On s'attend à ce que les effets liés aux deux sources s'additionnent algébriquement.

Cette hypothèse générale doit être spécifiée en fonction des différents cas de figure suivants :

H2 : Dans le cas où les deux sources sont identifiées :

$a$ / Si elles sont toutes deux compétentes on devrait observer un renforcement de la prise en compte du présupposé. Ce qui devrait se traduire par un jugement de vérité Iorsqu'il y a coorientation entre le posé et le présupposé (verbes factifs) et par un jugement d'incertitude, lorsqu'il y a anti-orientation (verbes contrefactifs).

$b$ / Si elles sont toutes deux non compétentes on devrait observer une minimisation de la prise en compte du présupposé, ce qui devrait se traduire par un jugement d'incertitude dans les deux cas verbes factifs et verbes contrefactifs.

c/ Si seule l'une d'entre elles est reconnue compétente : s'il s'agit du locuteur, on devrait observer une maximisation de la prise en compte du présupposé. Ce qui devrait se traduire par un jugement de vérité lorsqu'il y a utilisation d'un verbe factif et par un jugement de fausseté lorsqu'il y a utilisation d'un verbe contrefactif.

Par contre, s'il s'agit de l'énonciateur on devrait observer une minimisation de la prise en compte du présupposé. Ce qui devrait se traduire par un jugement d'incertitude (voire mème de vérité) et utilisation d'un verbe factif ou d'un contrefactif :

H3 : Dans le cas où seule l'une des deux sources est identifiée. si celle-ci est compétente on devrait observer des effets analogues à ceux décrits dans $\mathrm{H} 2 c$; par contre si celle-ci est non compétente on devrait observer une minimisation de la prise en compte du présupposé ce qui devrait se traduire dans tous les cas par un jugement d'incertitude. 


\section{CONSTRUCTION DU MATERRIEL EXPERIMENTAL}

\section{LES VERBES TESTÉS}

Nous testerons les trois catégories de verbes ci-dessus décrites : les verbes factifs, les verbes contrefactifs et les verbes non factifs, mais nous ne retiendrons que 3 verbes au sein de chaque catégorie, à savoir :

- (pour les verbes factifs) : SAvoir, SE DOUTER, REgRETTER;

- (pour les verbes contrefactifs) : PRÉTENDRE, S'IMAGINER, SE FIGURER ;

- (pour les verbes non factifs) : croire, penSER, Craindre.

CONSTRUCTION DES PHRASES PROPOSÉES AUX SUJETS EXPÉRIMENTAUX

Nous proposons donc de tester l'appréhension, par des sujets expérimentaux, du posé et du présupposé véhiculés par ces trois types de verbes insérés dans des phrases qui seront construites en faisant varier systématiquement :

1) la catégorie du verbe ;

2) la compétence de la source A ;

3) la compétence de la source $B$.

Les phrases proposées aux sujets expérimentaux auront la forme générale suivante :

$A$ vous dit " B V y "

où A sera la source $A$ (ou locuteur);

B la source B (ou énonciateur);

$V$ le verbe modal (qui pourra être un verbe factif, un contrefactif, ou un non factif);

$y$ l'événement à juger (référent).

L'expérimentation fera varier systématiquement :

1) les catégories de verbes :

Verbes factifs, contrefactifs et non factifs.

2) la compétence de la source A et de la source B.

Soit les cas de figure suivants :

Source A compétence (?) / source B compétence (?)

Source A compétence (?) / source B compétence (+)

Source A compétence (?) / source B compétence (-)

Source A compétence ( $+1 /$ source B compétence (?)

Source A compétence $(t) /$ source B compétence $(+)$

Source A compétence $(+) /$ source B compétence $(-)$

Source A compétence (-)/ source B compétence (?)

Source A compétence $(-)$ / source B compétence (+)

Source A compétence $(-) /$ source B compétence (-) 
où l'on a compétence (?) lorsque la compétence de la source n'est pas précisée;

compétence $(+)$ lorsque la source s'avère compétente ;

et compétence $(-)$ lorsque la source s'avère non compétente.

Chaque groupe expérimental permettant de tester une catégorie de verbes. Chaque sujet expérimental ayant à juger 27 phrases présentées dans un ordre aléatoire (pour une liste détaillée des items, cf. annexe).

\section{LE PLAN EXPERIMENTAL}

\section{Les variables considerées}

Variables indépendantes :

Catégorie de verbes testée : facteur à trois modalités (verbes factifs, verbes contrefactifs, verbes non factifs), ce qui déterminera les trois groupes expérimentaux;

- Compétence de la source $A$ : facteur à trois modalités $(C(?), G(+)$, $\mathrm{C}(-))$;

- Compétence de la source B : facteur à trois modalités $(\mathrm{C}(?), \mathrm{C}(+)$, $\mathrm{G}(-))$;

- Le nombre de verbes testé dans chaque catégorie.

Variable dépendante : Degré de vérité/fausseté accordé par les sujets à l'événement $y$.

Soit le plan expérimental suivant :

$$
\mathrm{S}^{\mathbf{1 5}}\left\langle\mathrm{G}^{3}\right\rangle * \mathrm{CA}^{3} * \mathrm{CB}^{3} * \mathrm{~V}^{3}
$$

où $\mathrm{S}$ désigne le nombre de sujets par groupe;

G désigne le nombre de groupes, chaque groupe permettant de tester une catégorie de verbes (factifs, contrefactifs, non factifs); CA désigne la compétence de la source $\mathrm{A}$ : facteur à 3 modalités (compétence (?) compétence (+) compétence $(-)$ );

$\mathrm{CB}$ désigne la compétence de la source $\mathrm{B}$ : facteur à 3 modalités (compétence (?) compétence (+) compétence (-1) ; et $\mathrm{V}$ désigne le nombre de verbes testés par catégorie (3).

\section{Les sujets}

45 sujets volontaires ayant entre 20 et 25 ans, étudiants en premier cycle à l'Université, de langue native française ont été retenus.

\section{Les consignes}

- Chaque sujet recevait un livret expérimental comportant 28 feuillets. Sur le premier de ces feuillets était inscrite la consigne suivante : "Je vais vous présenter une série de phrases. Chacune de ces phrases 
sera suivie d'une proposition qui explicitera ou tirera une conséquence à partir des informations contenues dans la phrase présentée.

"Sous cette proposition se trouvera une échelle en 7 points allant de tout à fait faux à tout à fait vrai.

"Je vous demanderai d'entourer un des points de l'échelle sachant que si vous entourez le 1 cela signifie que vous pensez que la phrase proposée est tout à fait fausse, et que si vous entourez le 7 cela signifie que vous pensez qu'elle est tout à fait vraie. Les notes intermédiaires vous permettront de moduler votre jugement. *

\section{Exemple :}

Pierre vous dit : "Jacques fait semblant d'être malade. "

Selon vous cette phrase : "Jacques est malade ", est-elle vraie ou fausse? (vous donnerez votre réponse en entourant un des points de l'échelle suivante).

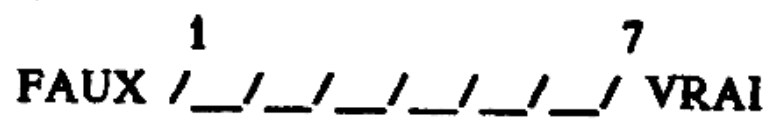

- Après que les sujets aient lu et compris la consigne, l'expérimentateur leur proposait de tourner la page suivante. Chacune des 27 pages suivantes comportait (chaque page contenait un item à juger) :

1) une phrase dont la forme générale était :

$A$ vous dit " $B$ verbe $y$ ";

2) une proposition inférence correspondant à l'affirmation de l'événement " $y$ ";

3) une consigne demandant au sujet d'attribuer un degré de vérité à l'événement " $y$ » en entourant un des points de l'échelle.

Par exemple aux phrases de la forme :

A vous dit " $\mathrm{B} \mathrm{X}$ la récolte est perdue " étaient associées la consigne et la proposition inférence suivantes :

Selon vous cette phrase : "La recolle est perdue ", est-elle vraie ou fausse? (vous donnerez votre réponse en entourant un des points de l'échelle suivante).

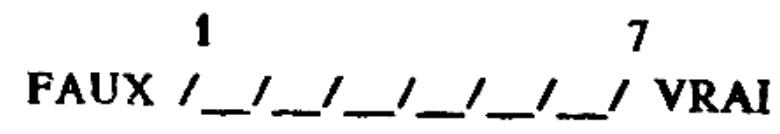

\section{RÉSULTATS}

Les tableaux présentés ci-dessous sont des tableaux globaux dans lesquels ne figurent pas les "notes "attribuées par les sujets, verbe par verbe. 
(Nous prenons la liberté d'utiliser ce terme afin d'éviter d'alourdir le texte par l'emploi trop fréquent de la périphrase " degré de vérité/fausseté accordé à l'événement $y$ ". Ce choix se justifie de par le fait que ce degré de vérité/fausseté se concrétise par une " note " allant de 1 à 7 sur l'échelle de mesure.)

Nous avons en effet regroupé les verbes testés au sein de leurs catégories respectives : factifs, contrefactifs et non factifs. Ceci a été possible, car il n'y avait pas de différences significatives entre les notes attribuées par les sujets au sein de chaque catégorie de verbe (excepté dans la catégorie des factifs entre le verbe " se douter " et le verbe "savoir " d'une part $(l=4,15$; $p<.01)$ et entre ce même verbe et le verbe " regretter " $(t=4,15$; $p<.01)$ )

TABleaU IA. - (Comparaisons inter-groupes.) Tableau mettant en évidence les effets du facteur: "compétence de la source" ( $A$ et $B)$. Les chiffres contenus dans les cases sont obtenus par simple moyennage des notes données par les sujets (notes se situant sur une échelle allant de 1 à 7 )

(Between groups comparisons.) This table displays effects of the factor : "competence of the source" (A and B). The data in the cells are obtained by taking an average of subjects' scores on the attitude scale (ranging from 1 to 7 )

\begin{tabular}{|c|c|c|c|c|}
\hline CA & $C B$ & $\begin{array}{r}\text { G1 } \\
\text { FACTIFS }\end{array}$ & $\begin{array}{r}\text { G2 } \\
\text { c. FACTIFS }\end{array}$ & $\begin{array}{c}\text { G3 } \\
\text { N.FACTIFS }\end{array}$ \\
\hline CA? & CB? & $5.10=$ & 2.57 & $2{ }^{93}$ \\
\hline CA? & $\mathrm{CB}+$ & 5.95 & 4.31 & 4.66 \\
\hline$C A$ ? & $\mathrm{CB}-$ & $4.13=$ & 2.95 & 2.64 \\
\hline CAt & $\mathrm{CB}$ ? & 5,08 & 2.77 & 3.11 \\
\hline $\mathrm{CA}+$ & $\mathrm{CB}+$ & 5.59 & 4.44 & 4.06 \\
\hline CAt & $\mathrm{CB}-$ & 450 & 3.66 & 2.93 \\
\hline $\mathrm{CA}-$ & $C B$ ? & 4.26 & 2.95 & 2.86 \\
\hline $\mathrm{CA}-$ & $\mathrm{CB}+$ & 5.50 & 5.22 & 4.62 \\
\hline $\mathrm{CA}-$ & $\mathrm{CB}-$ & 4.48 & 2.93 & 2.86 \\
\hline
\end{tabular}


TABleaU IB. - (Comparaisons intra-groupes.) Tableau mettant en évidence les effets du facteur compétence de la source ( $A$ et $B$ )

(Within groups comparisons.) This table displays effects of the factor " competence of the source" (A and B)

\begin{tabular}{|c|c|c|c|c|}
\hline CA & $C B$ & $\begin{array}{r}\text { G1 } \\
\text { FACTIFS }\end{array}$ & c. FACTIFS & $\begin{array}{c}\text { G3 } \\
\text { N.FACTIFS }\end{array}$ \\
\hline CA? & $\mathrm{CB} ?$ & $\mathrm{rf}^{5.10}$ & $\Gamma^{2.57}$ & $\Gamma^{2.93}$ \\
\hline CA? & $\mathrm{CB}+$ & 5.95 & 4.31 & 4.66 \\
\hline$C A ?$ & $\mathrm{CB}-$ & {$\left[L_{4.13}\right.$} & $L_{2.95}$ & L 2.64 \\
\hline $\mathrm{CA}+$ & $\mathrm{CB}$ ? & 5.08 & $\int[2.77$ & 3.11 \\
\hline $\mathrm{CAt}$ & $\mathrm{CB}+$ & 5.59 & 4.44 & 4.06 \\
\hline $\mathrm{CA}+$ & $\mathrm{CB}-$ & 4.50 & $L L 3.66$ & [2.93 \\
\hline $\mathrm{CA}-$ & $\mathrm{CB} ?$ & 4.26 & 2.95 & 2.86 \\
\hline $\mathrm{CA}-$ & $\mathrm{CB}+$ & 5.50 & 5.22 & 4.62 \\
\hline $\mathrm{CA}-$ & $\mathrm{CB}-$ & -4.48 & {$[2.93$} & $L_{2.86}$ \\
\hline
\end{tabular}

Note s lorsque $p<=.05$

Légende concernant les deux tableaux $1 \mathrm{~A}$ et 18 :

CA? = La compétence de la source A (locuteur) n'est pas précisee.

$\mathrm{CA}+\mathrm{La}$ source $\mathrm{A}$ est compétente

$C A-=$ La source $A$ est non compétente cisée.

$C B$ ? = La competence de la source $B$ (énonciateur) n'est pas pré-

CB+ = La source B est compétente

$C B-=L a$ source $B$ est non compétente

Factifs = Verbes de la categorie des Factifs

c. Factifs = Verbes de la categorie des Contrefactifs

N. Factifs = verbes de la catégorie des Non Factifs

COMMENTAIRE DES RÉSULTATS

Lorsqu'on observe les comparaisons, faites à l'aide du $t$ de Student (groupes indépendants), données dans le tableau IA, on note que toutes les comparaisons effectuées entre les groupes 1 
et 3 sont significatives. Il en est de même pour celles qui concernent les groupes 1 et 2 (excepté pour les conditions $\mathrm{CA}+/ \mathrm{CB}-$ et $\mathrm{CA}-(\mathrm{CB}+)$.

Le phénomène le plus marquant réside dans le fait que l'on n'observe pas de différences significatives entre les " notes " données par les sujets des groupes 2 et 3 (et ce quelle que soit la structure de la phrase-test proposée). Il semblerait ainsi que les sujets de ces deux groupes attribuent systématiquement le même degré de vérité/fausseté à l'événement $y$ présenté dans les phrases test, que ce dernier soit introduit par un verbe contrefactif (donc présuppositionnel) ou par un verbe non faclif (donc non présuppositionnel).

A partir de ce constat, trois hypothèses explicatives sont envisageables :

Première hypolhèse : Les sujets expérimentaux du groupe 2 n'ont pas appréhendé le présupposé contenu dans les verbes contrefactifs. Ils ont donc traité ces verbes comme s'ils étaient non présuppositionnels, ce qui expliquerait la similarité des réponses des groupes 2 et 3.

Deuxième hypothèse : Les sujets expérimentaux du groupe 3 ont spontanément (la cause reste encore à déterminer) doté les verbes non factifs d'un élément présupposé analogue à celui véhiculé par les verbes contrefactifs, ce qui serait à l'origine de ce traitement équivalent.

Troisième hypothèse : Il faut reconsidérer cette classification trop rigide et admettre que le critère linguistique n'est pas le seul qui soit pris en considération : entrent également en ligne de compte des variables d'ordre extra-linguistique.

$\mathrm{Au}$ vu des résultats contenus dans le tableau IB il semble que de ces trois hypothèses il faille retenir la dernière.

A l'appui de cette thèse trois faits doivent être notés :

1) On remarque une différence significative (au sein de chaque groupe expérimental) lorsqu'on fait varier la compétence de la source A (locuteur) et de la source $B$ (énonciateur) dans les phrases test proposées aux sujets expérimentaux. Ce qui nous amène à rejeter la première hypothèse. 
En effet, si cette dernière s'avérait correcte, on n'observerait pas une telle différence. Car si les verbes contrefactifs étaient traités comme des non-factifs, cela entraînerait un regroupement systématique des "notes " au milieu de l'échelle de mesure (traduisant ainsi un degré d'incertitude attribué par les sujets à l'événement $y$ ) et ce quelle que soit la compétence de la source $A$ (locuteur) et de la source B (énonciateur).

2) De la même manière, on sera amené à rejeter la deuxième hypothèse, car si celle-ci se révélait exacte, nous observerions un regroupement systématique des " notes" vers la gauche de l'échelle de mesure.

3) A l'appui de la troisième hypothèse : l'examen du F obtenu à partir de l'analyse de la variance permet de mettre en évidence un effet global du facteur compétence de la source. Toutefois il s'agit ici de la compétence de la source $B(F(2,84)$ $=56,62 ; p>.001)$ et non de la source $A(F(2,84)$ $=0,27, \mathrm{NS}$.

A l'appui de ceci, si l'on considère plus précisément les trois groupes expérimentaux (tableau IA) on remarque que le degré de vérité accordé à l'événement $y$ augmente significativement lorsque l'énonciateur (B) est reconnu compétent $(\mathrm{CB}+$ ) (nous noterons à ce propos que le facteur compétence de l'énonciateur (B) détermine à un tel point les réponses des sujets expérimentaux que l'on n'observe pas de différences significatives entre les trois groupes lorsqu'on observe la condition $\mathrm{CA}-\mathrm{CB}+$ : tous les sujets jugent l'événement globalement vrai).

Il apparaitrait donc qu'il faille nuancer nos hypothèses de départ, selon lesquelles le facteur "compétence de la source" a un effet déterminant, et préciser qu'il s'agit non pas de la compétence du locuteur (A) mais de la seule compétence de l'énonciateur (B).

Ces dernières remarques étant par ailleurs confirmées par le tableau II, ci-dessous, qui regroupe les "notes " en fonction du facteur " compétence de la source A ".

On note en effet, au vu du tableau II dans lequel sont données les comparaisons faites grâce au $t$ de Student, qu'il n'y a pas de différences significatives (à l'intérieur de chaque groupe) entre les conditions $\mathrm{CA}$ ?, $\mathrm{CA}+$, et $\mathrm{GA}$ -

Les comparaisons intergroupes confirment le fait que seules les comparaisons entre les groupes 1 et 2 sont significatives. 
TABLEAU II. - Tableau mettant en évidence les effets du facteur : "compétence de la source A ". Les chiffres contenus dans les cases sont obtenus par simple moyennage des notes données par les sujets (notes se situant sur une échelle allant de $1 \grave{a} 7$ )

This table displays effects of the factor : " competence of the source $A$ ". The data in the cells are obtained by taking an average of subjects' scores on the attitude scale (ranging from 1 to 7 )

\begin{tabular}{|c|c|c|c|c|}
\hline CA & $\mathrm{CB}$ & $\begin{array}{r}\text { G1 } \\
\text { FACTIFS }\end{array}$ & G2 & N.FACTIFS \\
\hline & $\mathrm{CB}$ ? & & & \\
\hline \multirow[t]{3}{*}{ CA? } & $\mathrm{CB}+$ & 5.04 & 3.27 & 3.40 \\
\hline & $\mathrm{CB}-$ & & & \\
\hline & $\mathrm{CB}$ ? & & & \\
\hline \multirow[t]{3}{*}{$\mathrm{CA}+$} & $\mathrm{CB}+$ & 5.08 & 3.62 & 3.33 \\
\hline & $\mathrm{CB}-$ & & & \\
\hline & $C B ?$ & & & \\
\hline \multirow[t]{2}{*}{$\mathrm{CA}-$} & $\mathrm{CB}+$ & 4.74 & 3.70 & 3.44 \\
\hline & $\mathrm{CB}-$ & & & \\
\hline
\end{tabular}

Legende du Tableautr. :

CA? = La compétence de la source A (locuteur) n'est pas précisée.

CAt = La source A est compétente

$C A-=$ La source $A$ est non compétente cisée.

CB? = La competence de la source B (énonciateur) n'est pas pré-

$\mathrm{CB}+=\mathrm{La}$ source $\mathrm{B}$ est competente

$C B-=$ La source B est non competente

Factifs = Verbes de la catégorie des Factifs

C. Factifs = Verbes de la catégorie des Contrefactifs

N. Factifs = Verbes de la categorie des Non Factifs

Enfin, le troisième tableau, qui permet de regrouper les résultats sans tenir compte du facteur " compétence de la source", met en évidence, comme nous l'avions noté lors de l'analyse du tableau I, une similarité des réponses données par les sujets des groupes 2 et $3(F(1,28)=0.08)$. 
TABleaU III. - Tableau global permettant de mettre en évidence les différences entre les groupes 1,2 el 3 et plus précisément les différences entre les catégories de verbes (Factifs, Contrefactifs, Non Factifs) et ce indépendamment du facteur "compétence de la source " $A$ et $B$

This global table displays differences between the three groups and more specifically between the different verbs (Factive, counter factive, and non-factive) and this irrespective of the factor " competence of the source » $A$ and $B$

\begin{tabular}{|c|c|c|c|}
\hline & G1 & G2 & G3 \\
& FACTIFS & C.FACTIFS & N.FACTIFS \\
\hline$A * B * V$ & 4.95 & 3.53 & 3,40 \\
\hline
\end{tabular}

\section{DISCUSSION}

L'analyse des résultats permet de mettre en évidence le fait que les verbes factifs et contrefactifs ne peuvent être décrits uniquement à partir de critères strictement linguistiques.

Entrent également en jeu des variables d'ordre extra-linguistique comme, nous en avons eu un exemple ici, la compélence attribuée à l'énonciateur par le destinataire.

Toutefois nous retiendrons le comportement particulier des sujets expérimentaux du groupe 3 . En effet, nous avions noté (lors de l'analyse du tableau IB) que ces derniers attribuent (tout comme les sujets des groupes 1 et 2 ) un degré de vérité élevé à l'événement $y$ lorsque la source $B$ est compétente ( $C B+$ ).

Nous remarquons que les sujets de ce groupe sont les seuls á privilégier syslémaliquement cette condition $(\mathrm{CB}+)$ et à considérer les deux autres conditions (CB - et CB?) comme étant strictement équivalentes. En effet, nous avons toujours $\mathrm{CB}+>\mathrm{CB}$ ? $=\mathrm{CB}-$ quelle que soit la compétence de A (alors que pour les deux autres groupes expérimentaux, nous observons dans certains cas (conditions CA? (pour le groupe 1) et conditions $\mathrm{CA}+$ (pour le groupe 2)) une hiérarchisation entre CB? et $\mathrm{CB}$ -

Ces dernières remarques nous permettent de faire l'hypothèse selon laquelle les sujets font appel à deux stratégies de décision 
lorsqu'ils doivent juger de la vérité/fausseté de l'événement $y$. La première stratégie consiste à surinvestir la compétence de l'énonciateur, alors que la deuxième stratégie se fonde sur la prise en compte :

1) du facteur compétence de l'énonciateur jugement qui est modulé en fonction;

2) des facteurs : "type de verbe " et " compétence du locuteur ".

Le choix de la première stratégie, adoptée par les sujets du groupe 3, est déterminé par le fait que ces sujets ne possèdent que des informations d'ordre extra-linguistique (compétence de la source) pour émettre leur jugement. Le choix de la deuxième stratégie, adoptée par les sujets des groupes 1 et 2 , repose sur le fait que ces sujets ont en leur possession des informations d'ordre linguistique (utilisation d'un Verbe Factif ou d'un Contrefactif) et extra-linguistique (compétence de la source).

\section{CONCLUSION}

Il apparait au terme de cet article que l'on ne peut aborder l'étude de la compréhension sans considérer les informations, tant linguistiques qu'extra-linguistiques, prises en compte par le sujet. De ce point de vue, comprendre un énoncé, ce n'est pas - ou pas seulement - appréhender des signifiés linguistiques, c'est interpréter, ou plus exactement, c'est faire usage de ses divers savoirs et compétences afin d'accéder à une signification.

Ce que nous avons essayé de mettre en évidence, ici en ce qui concerne ce type de verbes, c'est le fait que l'on peut révéler un certain " degré de liberté de l'auditeur " dans l'appréhension des énoncés véhiculant des présupposés, car ce dernier opère un choix parmi l'ensemble des interprétations possibles et non pas un simple décodage des informations linguistiques qui lui sont fournies. En d'autres termes il interprète les significations. Cette interprétation se fondant sur des informations d'ordre linguistique mais aussi extra-linguistique (i.e. ses propres savoirs et compétences, ainsi que sur les savoirs et compétences qu'il prête à l'énonciateur). 


\section{ANNEXE}

Construction du materiel expérimental (le premier verbe correspond au groupe G1, les deux autres entre parenthèses correspondent respectivement aux groupes G2 et G3)

$1^{\text {er }}$ groupe d'items : CA? / CB?

Item 1 :

G1 (G2, G3) : Pierre vous dit : " Jacques satt (PRÉtend, GrotT) que la récolte est perdue."

\section{Item 2 :}

G1 (G2, G3) : René vous dit : "Serge se doute (s'imagine, pense) que le temps va changer."

\section{Item 3 :}

G1 (G2, G3) : Jean vous dit : "Paul regrette (se figure, craint) que le Vésuve entre en éruption. "

$2^{\mathrm{e}}$ groupe d'items : $\mathrm{GA}$ ? / $\mathrm{CB}+$

\section{Item 4 :}

G1 (G2, G3) : Pierre vous dit : " Jacques qui est ingénieur agricole SaIt (PRÉTBND, GRoIt) que la récolte est perdue."

\section{Item 5 :}

G1 (G2, G3) : René vous dit : "Serge qui est météorologue se doute (s'imagine, pense) que le temps va changer."

Item 6 :

G1 (G2, G3) : Jean vous dit : "Paul qui est volcanologue ReGrette (se figure, craint) que le Vésuve entre en éruption."

$3^{\mathrm{e}}$ groupe d'items : $\mathrm{CA}$ ? / $\mathrm{CB}-$

\section{Item 7 :}

G1 (G2, G3) : Pierre vous dit : "Jacques qui est météorologue sAIT (PRÉTEND, CROIT) que la récolte est perdue."

Item 8 :

G1 (G2, G3) : René vous dit : "Serge qui est volcanologue SE DOUTE (s'imagine, PENSE) que le temps va changer."

Itemp 9 :

G1 (G2, G3) : Jean vous dit : " Paul qui est ingénieur agricole RE(GRETTE (SE FIgURE, CRATNT) que le Vésuve entre en éruption." 
$4^{\circ}$ groupe d'items : $\mathrm{CA}+/ \mathrm{CB}$ ?

Item 10 :

G1 (G2, G3) : Pierre qui est ingénieur agricole vous dit : "Jacques SAIT (PRETEND, GROIT) que la récolte est perdue."

Item 11 :

G1 (G2, G3) : René qui est météorologue vous dit: "Serge se doute (S'IMAGINe, PENSE) que le temps va changer. "

Item 12 :

G1 $(G 2, G 3)$ : Jean qui est volcanologue vous dit: "Paul regrette (se pigure, craint) que le Vésuve entre en éruption."

$5^{e}$ groupe d'items : $\mathrm{CA}+/ \mathrm{CB}+$

Item 13 :

G1 (G2, G3) : Pierre qui est ingenieur agricole vous dit : "Jacques, un de mes collegues, sait (PREtend, crolt) que la récolte est perdue.

Item 14 :

G1 $(\mathrm{G} 2, \mathrm{G3})$ : René qui est météorologue vous dit : "Serge, un de mes collègues, se doute (''imagine, PENSE) que le temps va changer."

Item 15 :

G1 (G2, G3) : Jean qui est volcanologue vous dit : " Paul, un de mes collègues, regrette (SE FIguRE, craint) que le Vésuve entre en éruption. "

$6^{\mathrm{e}}$ groupe d'items : $\mathrm{CA}+/ \mathrm{CB}-$

\section{Item 16 :}

G1 (G2, G3) : Jacques, qui est ingénieur agricole, vous dit : " René qui est météorologue SAIT (PRÉTEND, cRoIT) que la récolte est perdue."

Item 17 :

G1 (G2, G3) : Serge qui est météorologue vous dit : " Jean qui est volcanologue se doute ('Imagine, PENSE) que le temps va changer."

Item 18 :

G1 (G2, G3) : Paul qui est volcanologue vous dit : "Pierre qui est ingénieur agricole regrette (SE pIGURE, craint) que le Vésuve entre en éruption." "

7 groupe d'items : $\mathrm{CA}-/ \mathrm{CB}$ ?

Item 19 :

G1 (G2, G3) : René qui est météorologue vous dit : "Jacques saIT (PRÉtexb, GROIT) que la récolte est perdue. " 
Item 20 :

G1 $(\mathrm{G} 2, \mathrm{G} 3)$ : Jean qui est volcanologue vous dit : "Serge sE DouTE (s'imagine, PENSE) que le temps va changer. "

Item 21 :

G1 (G2, G3) : Pierre qui est ingénieur agricole vous dit : " Paul REGRETTE (SE FIGURE, CRAINT) que le Vésuve entre en éruption."

$8^{\circ}$ groupe d'items : $\mathrm{CA}-/ \mathrm{CB}+$

Item 22 :

G1 $(\mathrm{G} 2, \mathrm{G3})$ : René qui est météorologue vous dit : " Jacques qui est ingénieur agricole saIt (PRÉtend, croIt) que la récolte est perdue."

Item 23 :

G1 (G2, G3) : Jean qui est volcanologue vous dit : "Serge qui est météorologue SE Doute (S'imaGine, PENSE) que le temps va changer."

\section{Item 24 :}

G1 (G2, G3) : Pierre qui est ingénieur agricole vous dit : " Paul qui est volcanologue regrette (SE figure, craint) que le Vésuve entre en éruption."

$9^{9}$ groupe d'items : $\mathrm{CA}-/ \mathrm{CB}-$

Item 25 :

G1 (G2, G3) : Pierre qui est météorologue vous dit : "Jacques, un de mes collègues, SAIT (PRÉTEND, CROIT) que la récolte est perdue."

Item 26 :

G1 (G2, G3) : René qui est volcanologue vous dit : Serge, un de mes collègues, SE doute (s'imagine, PENSE) que le temps va changer. "

Item 27 :

G1 (G2, G3) : Jean qui est ingénieur agricole vous dit : " Paul, un de mes collègues, Regrette (se figure, craint) que le Vésuve entre en éruption. "

\section{$R E ́ S U M E$}

De nombreuses distinctions entre factifs, contrefactifs, non-factifs ont été proposées sur la base de critères logiques ou linguistiques. Nous faisons l'hypothèse selon laquelle, dans les phrases contenant ces verbes suivis d'une proposition subordonné, les critères logiques ou linguistiques ne sont pas les seuls elements pris en compte par les sujets qui doivent juger de la vérité de l'événement contenu dans la proposition subordonnée. Entrent egalement en jeu des elements extra-linguistiques comme la competence de la source. Nous distinguons, dans l'usage indirect de ces verbes, deux sources différentes : le locuteur et l'énonciateur. 
Nous analysons l'apprèhension, par des sujets, du présupposé véhiculé par des verbes factifs et contrefactifs, en faisant varier la competence de chaque source. Les résultats mettent en évidence le fait que c'est la compétence de l'enonciateur qui joue un rôle déterminant, et ce au-delà des critères linguistiques.

Mots cles : verbes factifs, verbes non factifs, verbes contrefactifs, présupposition, compréhension du langage.

\section{BIBLIOGRAPHIE}

Day G., Caron J. - (1991) Le développement de la compréhension des verbes modaux " pouvoir " et * devoir " chez des enfants de six à treize ans, Archives de Psychologie, 59, 228, 55-69.

Ducrot 0. - (1972) Dire et ne pas dire, Paris, Hermann.

Ducrot $0 .-(1980)$ Les mots du discours, Paris, Editions de Minuit.

Fillmore C. J. - (1971) Verbs of judging : An exercise in semantic description, in C. J. Fillmore et D. T. Langendoen (Edit.), Studies in linguistic semantics, New York, Holt, Rinehart \& Winston, 273-290.

Grice H. P. - (1975) Logic and conversation in P. Cole (Edit.), Syntax and semantics, vol. 3 : Speech acts, New York, Academic Press, 41-51 (trad. franç. (1979) : Logique et conversation, Communications, 30, $57-72)$.

Harris R. J. - (1974) Memory and comprehension of implications and inference of complex sentences, Journal of Verbal Learning and Verbal Behavior, 13, 626-637.

Karttunen L. - (1971) Implicative verbs, Language, 47, 340-358.

Karttunen L. - (1973) La logique des constructions anglaises à complément. prédicatif, Langages, $30,56-80$.

Kiparsky P., Kiparsky C. - (1970) Facts, in M. Bierwisch et K. Heidolph (Edit.), Progress in linguistics, La Haye, Mouton, 1970, 143-173.

Lehalle H., Jouen F. - (1978) Quelques verbes d'opinion et leur présupposition : étude génétique de leur compréhension chez l'adolescent, Enfance, 4-5, 237-245.

Mac Namara J., Baker E., Olson C. L. - (1976) Four-years-olds understanding of pretend forget, and know : Evidence for propositional operations, Child Development, 47, 62-70.

Martin R. - (1983) Pour une logique du sens, Paris, PUF.

Martin R. - (1987) Langage et croyance : les univers de croyance dans la théorie sémantique, Bruxelles, Mardaga.

Oléron P., Legros S. - (1977) Présuppositions, implications linguistiques et atteinte de la signification de termes psychologiques par l'enfant, Journal de Psychologie Normale et Pathologique, 74, 409-429.

Oléron P., Legros S. - (1986) L'interprétation de verbes psychologiques factifs et non factifs par des enfants en fonction du contenu des énoncés, Cahiers de Psychologie Cognitive, 6, 545-562.

Zuber R. - (1977) Decomposition on factive, Studies in Language, 1 (3). 